\title{
Los Materiales de Cambio de Fase (MCF) empleados para la acumulación de energía en la arquitectura. Su aplicación en el prototipo Magic Box
}

\section{Phase Change Materials (PCMs) for energy storage in architecture. Use with the Magic Box prototype}

\author{
F. J. Neila González (*), C. Acha Román ${ }^{(*)}$, E. Higueras García(*) y C. Bedoya Frutos(*)
}

Recepción/Received: 11-X-06

Aceptación/Accepted: 10-I-07

Publicación online/Online publishing: 28-V-08

\section{RESUMEN}

El artículo muestra un sistema de acumulación de energía en Materiales de Cambio de Fase diseñado para un prototipo de vivienda con el que se han construido dos edificios bioclimáticos y autosuficientes. Estos edificios se han realizado en Madrid, Washington y Pekín. Se incluyen las características de estos materiales, de los sistemas constructivos donde se integraron, su valoración comparativa con los sistemas de acumulación sensibles y los resultados de la monitorización del edificio.

Palabras clave: propiedades físicas, temperatura, análisis térmico, parafina, materiales de cambio de fase.

\section{INTRODUCCIÓN}

El reciente Código Técnico de la Edificación desarrolla y aplica las exigencias que fija la Ley de Ordenación de la Edificación en aspectos de sostenibilidad y ahorro de energía, entre otros. EI DB HE1, Documento Básico de Ahorro de Energía basado en la reducción de la demanda, valora positivamente la concepción arquitectónica que aprovecha las captaciones solares pasivas en invierno y la ventilación nocturna en verano, reduciendo los valores exigidos a las transmitancias térmicas en las ventanas bien orientadas. Para que este aprovechamiento sea real y efectivo es necesario dotar al edificio de iner-

\section{SUMMARY}

The article shows an energy-accumulation system in change of phase materials, designed for a prototype dwelling used for building two bioclimatic and self-sufficient buildings. These bulidings have been built in Madrid, Washington and Beijing. The characteristics of these materials, the construction systems into which these materials were incorporated, its comparative valuation with sensitive accumulation systems, and the results of the building monitorization are included.

Keywords: physical properties, temperature, thermal analysis, parafine, phase change materials.

(*) Universidad Politécnica de Madrid (Madrid, España). 
cia energética. Sin embargo, estos sistemas funcionan aprovechando el calor sensible de su calentamiento o enfriamiento, lo que limita notablemente su capacidad de acumular energía, ya que nunca podrán enfriarse por debajo de la temperatura mínima del aire nocturno, ni calentar por encima de los límites que marca la energía solar captada en un corto número de horas. Por otro lado, el calentamiento o enfriamiento por conducción de los sólidos tiende a concentrar la energía en las capas más superficiales. Si bien el uso del agua resuelve ese inconveniente, por ser un fluido y aportar un mayor calor especifico, el límite de la temperatura sigue existiendo, siendo muy difícil que se superen los $20^{\circ} \mathrm{C}$ de cambio de temperatura. Otro de los inconvenientes de los sistemas de acumulación de energía en forma de calor sensible es que, tanto la carga, como la descarga de esa energía, se hacen a temperatura variable, ya que esa es la esencia fundamental de los sistemas sensibles, por lo que nunca funcionarán en condiciones constantes de bienestar.

Frente a este procedimiento de acumulación existe otro basado en el aprovechamiento del calor latente que corresponde (captar o ceder) a todos los materiales cuando cambian de estado. Esta característica es propia de todos los materiales, pero aquéllos en los que se puede aprovechar eficazmente se les denomina, de forma genérica, Materiales de Cambio de Fase (MCF), o, según sus siglas inglesas Phase Change Materials (PCM). Como es sabido, mientras que se está realizando el proceso, y hasta que no se completa, la temperatura a la que se realiza no varía. En sustancias puras, como el agua, esa temperatura es inalterable $\left(0^{\circ} \mathrm{C}\right.$ para la licuación-congelación), pero si esa estabilidad se quiere conseguir en mezclas deben ser eutécticas, es decir, con unas proporciones y características determinadas, para que también siga inalterada.

Dependiendo del material, ese calor latente podrá ser mayor o menor, por lo que se pueden seleccionar los más adecuados, pero la gran ventaja que aportan es que, tanto el proceso de carga de energía, calentamiento o enfriamiento, es decir, licuación o solidificación, como el de descarga, pasando al estado anterior, se hacen a temperatura constante. De tal modo, que si se ha seleccionado un material cuya temperatura de cambio de estado es la deseada para el acondicionamiento, todo el proceso se realizará en condiciones óptimas. Si se elige el material adecuado, tanto por su calor latente de cambio de estado, buscando valores altos, como por la temperatura a la que se produce, en el rango del confort, se puede reducir notablemente el volumen de acumulación y permitir su integración arquitectónica en sistemas prefabricados novedosos o en sistemas constructivos convencionales.

Los materiales y las soluciones constructivas que se describen a continuación se diseñaron para ser empleados como el sistema de acumulación de la energía térmica que iba a servir para el acondicionamiento de dos viviendas (según el mismo prototipo) construidas en Madrid, Washington y Pekín, con la denominación de Magic Box. Se trata del prototipo de una vivienda unifamiliar de $70 \mathrm{~m}^{2}$ diseñada para participar, con su construcción en Washington, en el evento Solar Decathlon 2005, organizado por el Departamento de Energía de los EE.UU. El objetivo de este evento era diseñar, construir y monitorizar en funcionamiento real una casa autosuficiente; la construcción se efectuó a lo largo de los meses de septiembre y octubre de 2005 en Washington. Durante el tiempo que permaneció la casa construida en EE.UU., se monitorizó, obteniéndose datos muy favorables del comportamiento de las sustancias de cambio de estado durante ese periodo de tiempo. La construcción en Pekín corresponde a la partición de este mismo edificio, representado a España, en el Future House, exhibición organizada por el Ministerio de la Construcción chino donde, representando a ocho países, se mostrará lo más significativo de la tecnología vinculada a la vivienda desarrollada en el mundo. En Madrid hay construido otro modelo del mismo prototipo, donde, hasta 2008, apoyados en el proyecto de investigación HELIODOMO, se tomarán datos de su comportamiento enérgico, haciendo modificaciones en las sustancias de cambio de estado que se describen en este artículo con el objeto de optimizar su integración arquitectónica y su comportamiento energético.

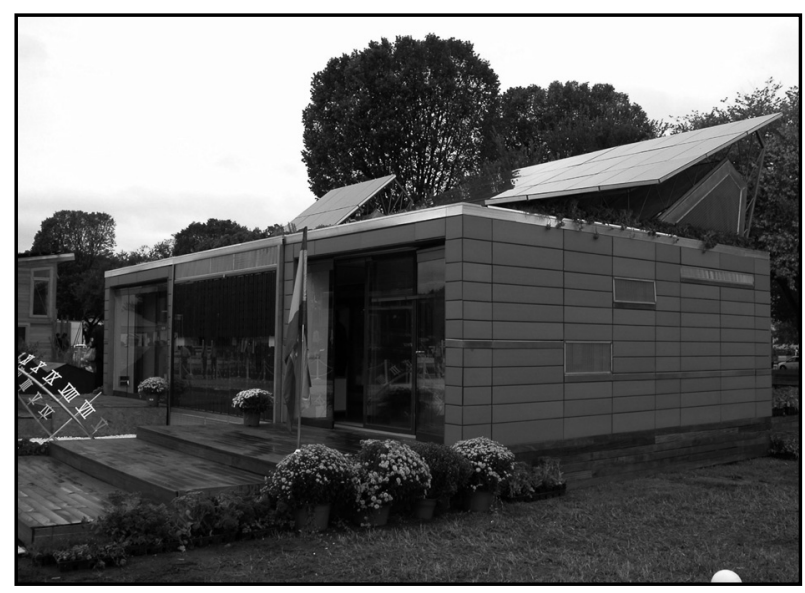

Figura 1. Foto de la vivienda durante el periodo en el que estuvo construida en Washington.

Este prototipo de viviendas se desarrolló abarcando premisas de habitabilidad, sostenibilidad y control de contaminación, energía, recursos y materiales. El proyecto tenía como objetivo fundamental la construcción de una vivienda autosuficiente en energía eléctrica, pero también bioclimática en su más amplia definición, por lo que el acondicionamiento debía ser pasivo, calentándose el edifico con radiación solar y cargas internas y refrigerándose con ventilación nocturna. Por ello era imprescindible 
el uso de un sistema de acumulación eficaz que descargará la energía a temperatura constante y asegurara, de ese modo, permanentemente las condiciones de bienestar determinadas en las bases del concurso de $23^{\circ} \mathrm{C}$. El material que se eligió fue una sustancia que cambia de estado a una temperatura controlada y en la que la energía que se acumula fuera la del calor latente de su cambio de fase.

La acumulación de cualquier tipo de energía renovable dentro de un edificio resulta muy compleja. Las formas clásicas de energías aprovechables en un edificio son la radiación solar para el calentamiento en invierno, y la ventilación nocturna en verano.

Ambos fenómenos están vinculados a las ventanas, por lo que es más fácil acumular la energía, calor o frío, cerca de ellas. En el caso concreto de la radiación, debido a la inclinación de los rayos solares, la incidencia natural es en el suelo, por tanto, el punto más eficaz para acumular la energía solar es el pavimento. Generalmente la acumulación en forma de calor sensible en los forjados es escasa, y prácticamente nula cuando se emplean recubrimientos de madera, ya que tienen un lentísimo calentamiento. De igual modo la ventilación nocturna, que es una masa de aire fresco a baja velocidad, una vez que penetra por la ventana, debido a su mayor densidad con respecto al aire caliente, tiende a desplazarse hacia el suelo depositándose como un ligero colchón sobre el pavimento. Por ello, parecía razonable depositar los materiales de cambio de fase (MCF) que fueran a emplearse en el suelo, ya fuera en su superficie o bajo ella.

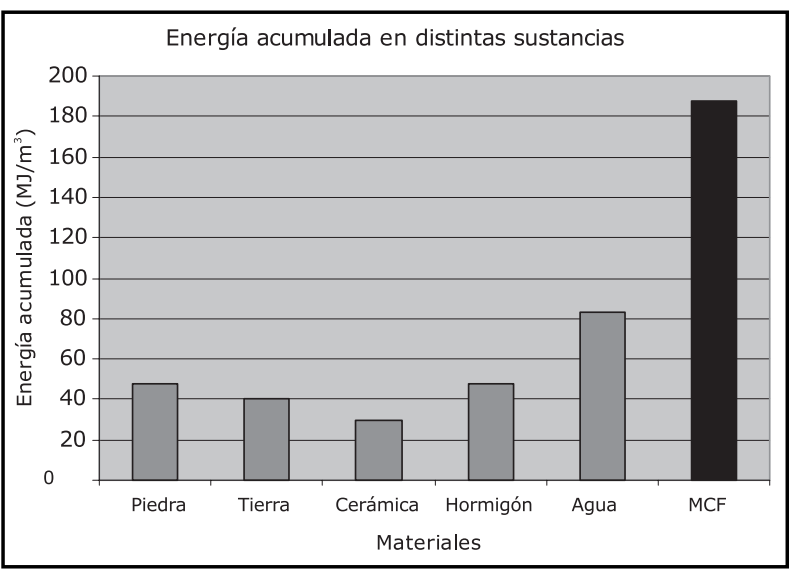

Figura 2. Comparación entre la energía acumulada en forma de calor sensible en varios materiales (variación de temperatura de $20^{\circ} \mathrm{C}$ ), en color gris, y la acumulada en forma latente en el Material de Cambio de Fase empleado en el edificio, en color negro.

La sustancia elegida para acumular la energía es un hidrocarburo en forma de parafina mezclada, que cambia de estado a $23^{\circ} \mathrm{C}$. El calor latente de cambio de estado es de $188 \mathrm{~kJ} / \mathrm{kg}\left(171 \mathrm{MJ} / \mathrm{m}^{3}\right)$. Eso quiere decir que es 6 veces más eficaz que acumular en forma de calor sensible en un muro de ladrillo de macizo al que hemos logrado incrementar su temperatura en $20^{\circ} \mathrm{C}$ (por ejemplo de 20 a $40{ }^{\circ} \mathrm{C}$ ). El calor específico del ladrillo es $0,84 \mathrm{~kJ} / \mathrm{kg} \cdot \mathrm{K}$ y su densidad $1800 \mathrm{~kg} / \mathrm{m}^{3}$, por lo que la energía acumulada en un metro cúbico es sólo 30,24 MJ. El sistema de calor sensible descarga la energía a temperatura variable, ya que se va enfriando o calentando en el proceso según se haya acumulado frescor o calor. Sin embargo, el sistema empleado basado en el cambio de fase se descarga siempre a $23^{\circ} \mathrm{C}$, hasta que cambie de estado por completo, momento en el que se procedería a un intercambio sensible.

\section{CARACTERÍSTICAS FÍSICO-QUÍMICAS DEL MATERIAL DE CAMBIO DE FASE Y LAS BAL- DOSAS EMPLEADAS}

\section{Características del material de cambio de fase:}

Composición ${ }^{1}$ :

Mezcla eutéctica de derivados de hidrocarburos saturados (hidrocarburo parafínico o parafina, $\mathrm{C}_{n} \mathrm{H}_{2 n+2}$ ) e insaturados o no saturados, con aditivos anticorrosivos, preservativos y colorantes.

Clasificación:

R: R22; R36/38

S: S37; S24/25

Conductividad térmica:

$0,232 \mathrm{~W} / \mathrm{m} \cdot \mathrm{K}$ (estado líquido)

$0,464 \mathrm{~W} / \mathrm{m} \cdot \mathrm{K}$ (estado sólido)

Densidad:

$1110 \ldots 1120 \mathrm{~kg} / \mathrm{m}^{3}$

Calor específico:

$836 \ldots 1672 \mathrm{~J} / \mathrm{kg} \cdot \mathrm{K}$

Difusividad térmica:

$0,25 \times 10^{6} \mathrm{~m}^{2} / \mathrm{s}$

Efusividad térmica:

$932 \mathrm{~s}^{1 / 2} \cdot \mathrm{W} / \mathrm{m}^{2} \cdot \mathrm{K}$

Calor latente de cambio de fase $188 \mathrm{~kJ} / \mathrm{kg}$

Temperatura de cambio de fase: $23^{\circ} \mathrm{C}\left( \pm 1^{\circ} \mathrm{C}\right)$

Temperatura de ignición: $>245^{\circ} \mathrm{C}$

Toxicidad y peligrosidad: Ningún riesgo en la inhalación Muy bajo riesgo en la ingestión Irritación cutánea tras un largo contacto

1 Compañía: ACUFRÍO, S.L. 


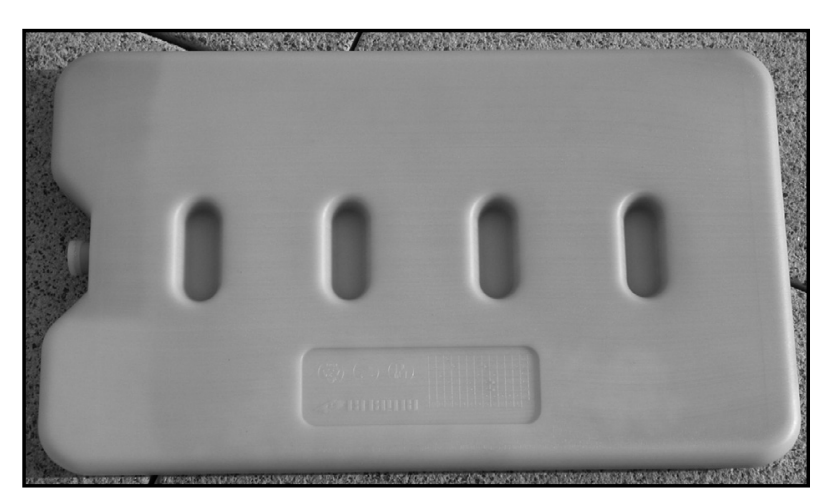

Figura 3. Cápsulas contenedoras de los MCF.

Uno de los grandes inconvenientes de emplear sustancias de cambio de estado en un edificio es su integración constructiva y arquitectónica. En este prototipo se integró en un falso suelo, a modo de suelo técnico, en dos capas de cápsulas de plástico de $3 \mathrm{~kg}$ y unas dimensiones de $28 \times 46 \times 3 \mathrm{~cm}$ cada una, con un separador de $2 \mathrm{~cm}$ entre ellas y entre ellas y el suelo. La baldosa de pavimento recoge la última capa de MCF. Se trata de una baldosa de gres sobre una base metálica para permitir su apoyo en las cuatro esquinas para formar el suelo técnico. Esa base metálica es un recipiente cerrado al que se puede acceder por unos orificios de llenado; en cada una se introdujeron $5 \mathrm{~kg}$ de parafina. El total de MCF instalados es de $1326 \mathrm{~kg}$. Con esa cantidad, y teniendo en cuenta los flujos de energía que se establecen no cambia de estado completamente en ningún momento, lo que asegurará que las descargas sean siempre a $23^{\circ} \mathrm{C}\left( \pm 1^{\circ} \mathrm{C}\right)$. Para las condiciones climáticas concretas del periodo de exhibición y monitorización en Washington, durante las horas de calor pueden realizarse 8,5 renovaciones de aire a la hora sin que se licue o congele por completo la parafina.

\section{Características de las baldosas empleadas:}

Placa de gres2:

Dimensiones:

$600 \times 600 \times 5 \mathrm{~mm}$

Resistencia térmica:

$$
0,30 \times 10^{-3} \mathrm{~m}^{2} \cdot \mathrm{K} / \mathrm{W}
$$

Difusividad térmica:

$$
>0,56 \times 10^{6} \mathrm{~m}^{2} / \mathrm{s}
$$

Contenedor metálico:

Altura:

$30 \mathrm{~mm}$, con una la relación de $10 \mathrm{~mm}$ de espesor por cada $2 \times 10^{6} \mathrm{~J} / \mathrm{m}^{2}$ de energía a acumular.

El contenedor metálico debe ser capaz de contener suficiente sustancia como para acumular toda la energía que reciba. Si es escasa cambiará completamente de estado

\footnotetext{
2 Compañía: ALCALAGRES, http://www.alcalagres.es.
}

y posteriormente comenzará a calentarse/enfriarse sensiblemente, perdiendo parte del interés de los sistemas de cambio de estado, descargar a temperatura constante. Por ello se aseguró una cantidad de sustancia mínima, en función de la energía a acumular. Esta última dependiendo del lugar, de la irradiancia solar, de la temperatura nocturna del aire, de las cargas internas, etc.
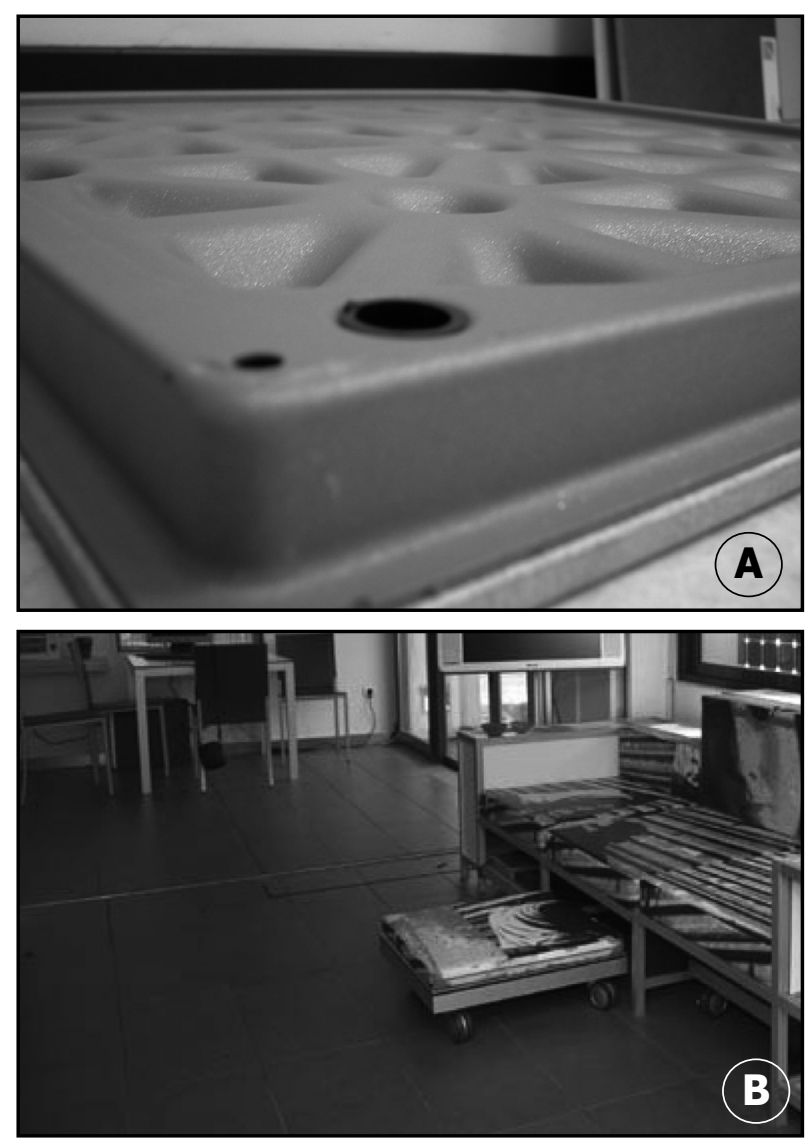

Figura 4. (A) Baldosa de pavimento vista por abajo, con el contenedor metálico y los orificios de llenado, y por arriba, colocada en la vivienda (B).

\section{DESCRIPCIÓN DEL FUNCIONAMIENTO DEL SISTEMA}

Si estamos en condiciones de invierno y los Materiales de Cambio de Fase situados en el pavimento se encuentran congelados, es decir, a una temperatura inferior a la de cambio de estado, el calor del sol comenzará a descongelar las sustancias hasta su licuación total, si la energía en relación con la masa de sustancias de cambio de estado es suficiente, o dejando una mezcla de sólido líquido si es insuficiente.

Si estamos en condiciones de invierno y las sustancias de cambio de estado situadas en el pavimento se encuen- 
tran en estado líquido, es decir, a una temperatura superior a la de cambio de estado, el frescor del aire nocturno comenzará a congelar las sustancias hasta su solidificación total, si la energía en relación con la masa de sustancias de cambio de estado es suficiente, o dejando una mezcla de sólido-líquido si es insuficiente.

A lo largo del resto del día la sustancia realiza el proceso contrario cediendo la energía acumulada, siempre a la temperatura de cambio de estado, que se ha hecho coincidir con la de bienestar.

Todas estas opciones, de enfriamiento, calentamiento o ventilación, se realizan mediante el accionamiento de dos conjuntos de compuertas, combinadas con rejillas, que adoptan posiciones diferentes.

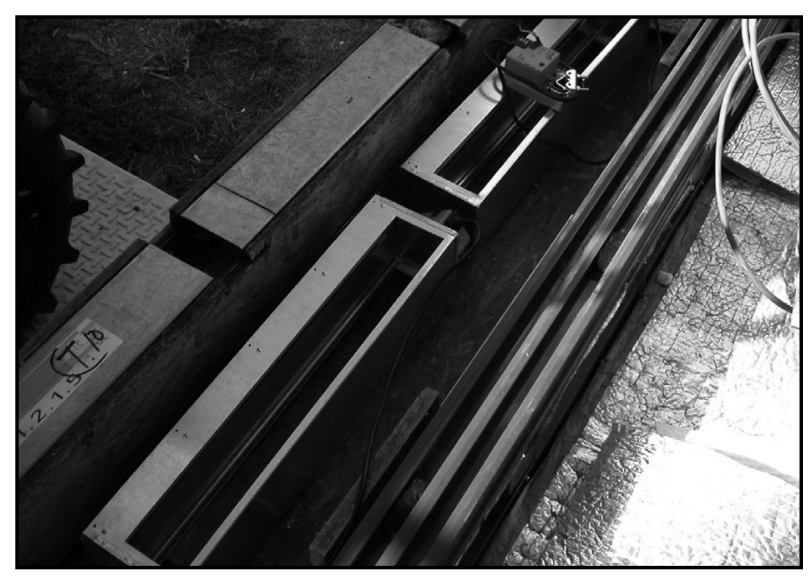

Figura 5. Compuertas de entrada y salida del aire.

El primer conjunto de compuertas las de toma de aire exterior o SUCCIÓN (fachada norte), pueden estar en posición captación o posición recirculación. En la primera posición se toma el aire exterior y envía al suelo técnico; corresponde a la captación del frescor nocturno (verano) o la toma de aire exterior para ventilación (verano o invierno). En la segunda posición permite la entrada al suelo técnico del aire interior (RECIRCULACIÓN), para mantener sus condiciones higrotérmicas (verano o invierno).

El segundo conjunto de compuertas, las de EXPULSIÓN (fachada sur), pueden estar en posición expulsión o posición recirculación. En la primera posición se expulsa el aire captado hacia el exterior, una vez que ha circulado por al suelo técnico; corresponde a la captación del frescor nocturno (verano). En la segunda posición permiten la salida del aire desde el suelo técnico hacia el interior de la vivienda (RECIRCULACIÓN) para acondicionarla (verano o invierno).

Una ventana o ventanal situado en un punto alto de la cubierta y a la altura del primer conjunto de compuertas (fachada norte), completa el procedimiento de ventilación para mejora de la calidad del aire interior.

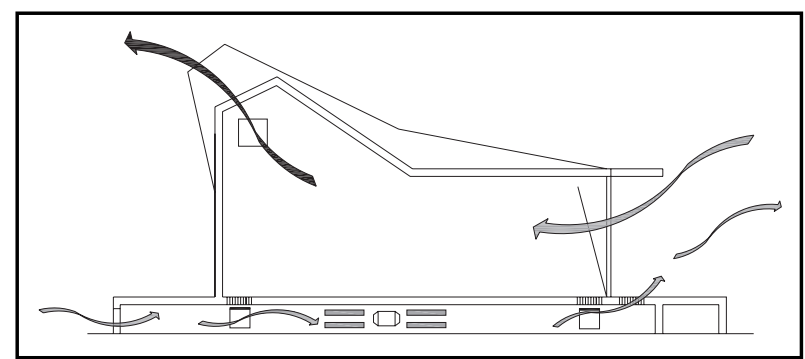

Figura 6. Esquema de funcionamiento en las noches de verano. Congelación gracias al frescor del aire nocturno. Posición CAPTACIÓN.

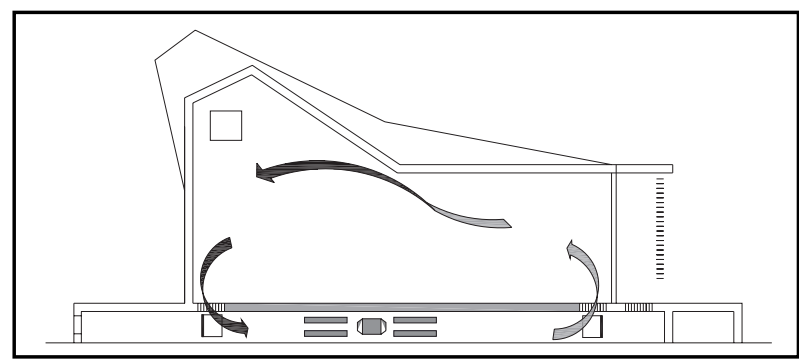

Figura 7. Esquema de funcionamiento en días de verano. Descarga del frescor acumulado en los MCF en el proceso de descongelación. Posición RECIRCULACIÓN interior.

El frescor que se capta lo aporta el aire de la noche cuando su temperatura baja lo suficiente. Para ello, se disponen de huecos de aspiración situados a la altura de un suelo técnico. Por ellos entra el aire durante las horas de la noche en las que el dispositivo inteligente detecte que las condiciones son adecuadas para su aprovechamiento energético. Si es así, se accionarán unos ventiladores de succión (12 ventiladores helicocentrífugos con un caudal de $169 \mathrm{~m}^{3} / \mathrm{h}$ cada uno) que hacen circular el aire por el suelo técnico expulsándolo por la fachada opuesta, una vez que han cedido su energía (frescor). Es la posición CAPTACIÓN de frescor.

Durante el día se recircula el aire interior de la vivienda por el suelo técnico, enfriándose al tiempo que cambian de estado los MCF de sólido a líquido y descargando el aire en la habitación a la temperatura de cambio de estado, es decir, $23^{\circ} \mathrm{C}$. Para proceder a esa recirculación las compuertas han adquirido la posición de RECIRCULACIÓN a través del interior de la casa.

El calor se capta básicamente de la radiación solar. El sol, calentando el interior del invernadero o incidiendo directamente sobre las baldosas de gres del suelo, aporta energía al aire. El sistema inteligente, al detectar esa 


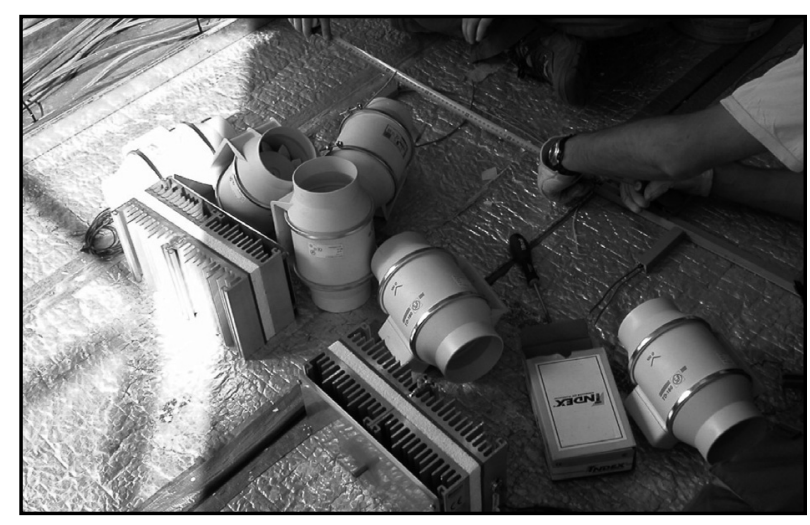

Figura 8. Ventiladores.

energía vuelve a accionar los ventiladores para llevar ese aire caliente al suelo técnico. En este caso lo hace a través de las rejillas situadas en el suelo en uno de los extremos de la vivienda. El aire, una vez que ha circulado y cedido su energía (calor), sale por las rejillas situadas en el suelo de la casa, pero en el otro extremo, con la intención de recircular de nuevo. Es la posición RECIRCULACIÓN.

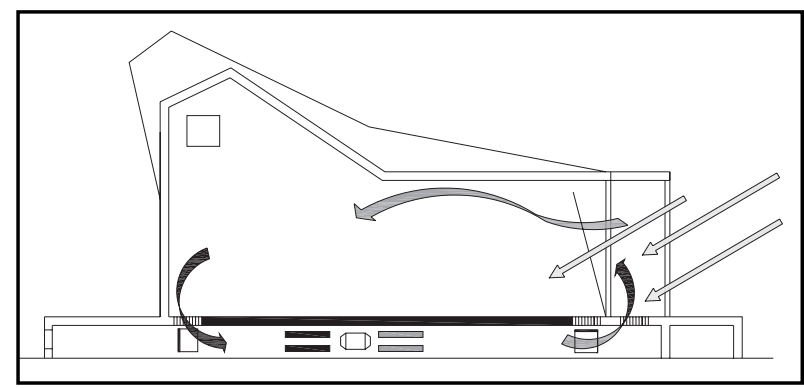

Figura 9. Esquema de funcionamiento en los días de invierno. Licuación de los MCF gracias al calor solar del invernadero, al que ha penetrado directamente en la vivienda y a las cargas internas. Posición RECIRCULACIÓN exterior.

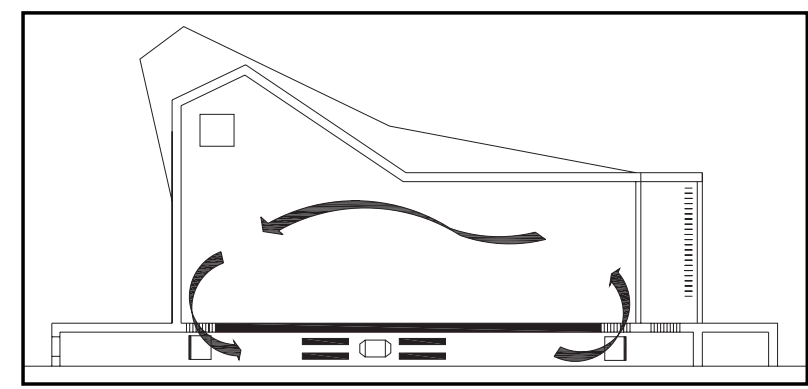

Figura 10. Esquema de funcionamiento en las noches de invierno. Descarga del calor acumulado en los MCF licuados. Posición RECIRCULACIÓN interior.

Durante la noche se recircula el aire interior de la vivienda por el suelo técnico, calentándose al tiempo que cambian de estado los MCF de líquido a sólido y descargando el aire en la habitación a la temperatura de cambio de estado, es decir, $23^{\circ} \mathrm{C}$. Para proceder a esa recirculación las compuertas han adquirido la posición de RECIRCULACIÓN a través del interior de la casa.

Si las condiciones de aire interior (contenido de sustancias contaminantes) no es el adecuado, el aire no se recirculará sino que se enviará al exterior procediendo a una entrada permanente de aire exterior. El aire entra al suelo técnico desde el exterior, aportando aire limpio, pasa por las sustancias acumuladores acondicionándose, penetra al interior de la vivienda por el segundo conjunto de compuertas, situadas en posición recirculación, y se expulsa, finalmente hacia el exterior, a través de las mencionadas ventanas abiertas.

Todos los dispositivos del sistema, los dos conjuntos de compuertas, en una u otra posición, los ventiladores, funcionando o apagados, los deshumidificadores, encendidos o apagados y las ventanas, abiertas o cerradas, se accionan a las órdenes del sistema inteligente, que relaciona las condiciones interiores con las exteriores.

\section{Posición de enfriamiento:}

- Cuando las condiciones exteriores son adecuadas para la acumulación de energía, es decir, temperatura exterior inferior a la de cambio de estado de los MCF acumuladores, y las condiciones interiores sean de bienestar, el sistema acciona los ventiladores y pone las compuertas en posición CAPTACIÓN. Cuando las condiciones del aire exterior dejen de ser favorables, es decir, la temperatura está por encima de la de cambio de estado de los MCF, se apagan los ventiladores.

- Cuando las condiciones interiores no son las de bienestar, es decir, con una temperatura superior, se analizan en primer lugar las condiciones exteriores. Si la temperatura exterior está en el entorno de la de bienestar ( \pm 1 ${ }^{\circ} \mathrm{C}$ ), se abren automáticamente las ventanas situadas a ambos lados de la casa para asegurar una ventilación natural cruzada. Si la temperatura exterior no cumple este requisito se accionan los ventiladores y se ponen las compuertas en posición RECIRCULACIÓN.

\section{Posición de calentamiento:}

- Cuando las condiciones exteriores son adecuadas para la acumulación de energía, es decir, incidencia de la radiación solar sobre la fachada captadora, o temperatura exterior superior a la de cambio de estado de los MCF acumuladores, o temperatura interior superior a la de bienestar (cargas internas), el sistema acciona los ventiladores y pone las compuertas en posición RECIRCULACIÓN.

- A pesar de que no se den ninguna de estas condiciones, los ventiladores siguen funcionado mientras la 
temperatura de impulsión por las rejillas de salida sea superior a la de succión por las rejillas de entrada.

\section{Posición de ventilación:}

- Si en cualquiera de las situaciones posibles mencionadas anteriormente la calidad del aire interior está por debajo del mínimo admisible, las compuertas y ventanas se ponen en posición VENTILACIÓN.

\section{CONCLUSIONES FINALES}

El edificio se ha monitorizado 3 en varios momentos para obtener datos del funcionamiento general de la vivienda y de la influencia de la masa térmica en la temperatura interior. El edificio es muy ligero, por tanto no aporta masa térmica suficiente como para estabilizar la temperatura interior en el rango del bienestar sin el empleo de sistemas mecánicos. Sin embargo, la masa térmica aportada por los MCF situados en el pavimento y en el suelo técnico resulta suficiente como para conseguir el objetivo deseado sin el empleo de sistemas mecánicos de acondicionamiento ni el gasto de energía adicional.

A continuación se muestran datos de la monitorización del edificio, tomados a lo largo de cuatro días, cuando estuvo en exhibición en Washington. Durante ese periodo de tiempo se mantuvieron los ventiladores apagados, de tal modo que el comportamiento fuera completamente pasivo y únicamente los MCF situados en el pavimento aportarán su masa térmica. Dado que las condiciones climáticas de los días siguientes fueron muy diferentes, no se podía realizar una comparación de los resultados haciendo funcionar a la vivienda con los ventiladores accionados. Por ello, a continuación se realizaron dos simulaciones para las mismas condiciones climáticas con el programa PETI, con los ventiladores en funcionamiento y en una situación teórica en la que se eliminaban las sustancias de cambio de estado de las baldosas de pavimento.

El resultado, como se puede ver, es muy aceptable en las condiciones medidas, aun sin emplear los ventiladores, ya que la temperatura máxima del periodo de cuatro días fue de $24,6^{\circ} \mathrm{C}$ y la mínima de $18,4{ }^{\circ} \mathrm{C}$, es decir, con una oscilación total de $6,2^{\circ} \mathrm{C}$. En el peor de los días la Oscilación Media Diaria (OMD) fue sólo de 4,4 ${ }^{\circ} \mathrm{C}$; a pesar de que mantener apagados los ventiladores implica reducir a casi una tercera parte la masa de MCF colocada en la vivienda. Todo ello demuestra la eficacia de estos Materiales de Cambio de Fase a la hora de aportar inercia térmica en un edificio (Figura 11).

Si funcionan los ventiladores, es decir, se aprovecha el $100 \%$ de la masa acumuladora dispuesta en el edificio, la temperatura interior se estabiliza aún más, alcanzándose

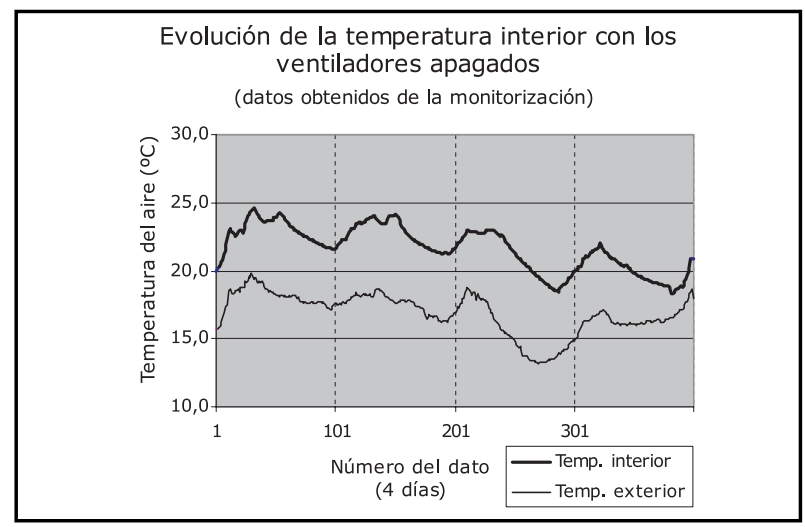

Figura 11.

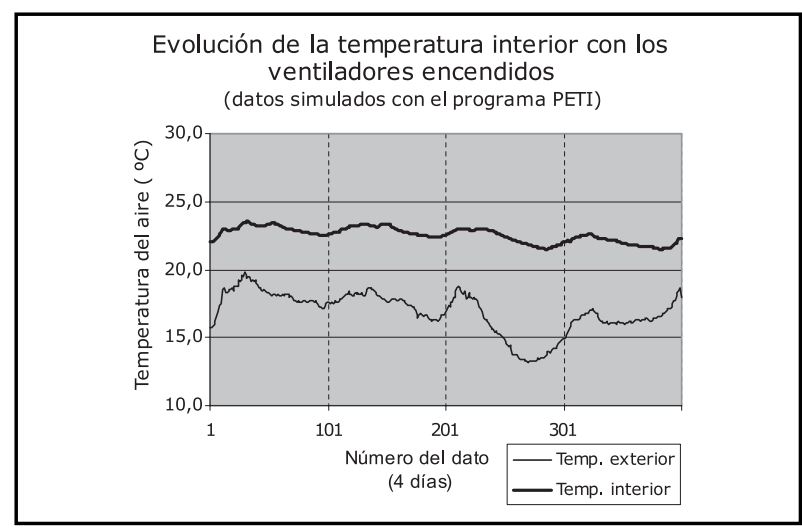

Figura 12.

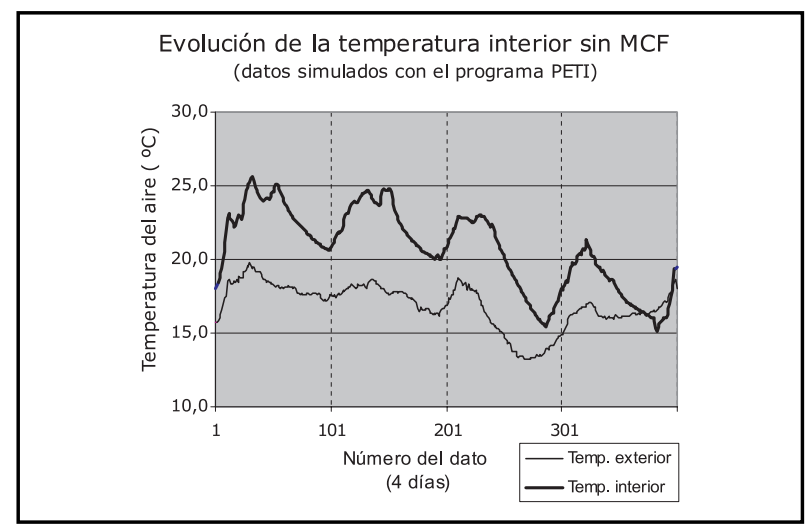

Figura 13.

una temperatura interior máxima en el periodo de los cuatro días de $23,5^{\circ} \mathrm{C}$ y una temperatura mínima de $21,4^{\circ} \mathrm{C}$, eso implica que la desviación máxima con respecto al objetivo fijado, que era mantener constate una temperatura de $23^{\circ} \mathrm{C}$ fue como máximo de $1,6^{\circ} \mathrm{C}$ (Figura 12).

Por el contrario, cuando se simula el funcionamiento de la vivienda sin los MCF, ni los de las baldosas ni los del 
suelo técnico, la línea de evolución de temperatura interior marcaría grandes fluctuaciones, ya que la casa, como se ha comentado es muy ligera, y no tiene más masa térmica que la que aportan los MCF (Figura 13).

Estas sustancias pueden aportar grandes beneficios energéticos a los edificios bioclimáticos que quieran reducir su dependencia energética para el acondicionamiento, cap- tando y acumulando la energía que aporta gratuitamente la naturaleza. Sus ventajas con respecto a los sistemas sensibles son evidentes, y sólo queda diseñar los sistemas constructivos que permitan su integración sencilla y económica en los edificios. En este prototipo se ha mostrado una opción posible, pero se está investigando en otras soluciones que también podrán usarse en el futuro.

\section{BIBLIOGRAFÍA}

(1) Ames, D.: The Past, Present and Future of Eutectic Salt Storage Systems, ASHRAE Journal, 31 (1989), pp. 26-28.

(2) Benard, C., Body Y., Zanoli A.: 1985. "Experimental Comparation of Latent and Sensible Heat Thermal Walls". Solar Energy vol. 34, Nº 6, pp. 475-487. Pergamon. http://dx.doi.org/10.1016/0038-092X(85)90021-0

(3) Cahn, R. W., Haasen, P., Kramer, E. J.: Materials Science and Technology, Volume 5: Phase Transformations in Materials, VCH (1991).

(4) Dincer, I. and Rosen, M. A.: Thermal Energy Storage. Systems and Applications. Wiley and Sons Ltd. West Sussex, UK. 2002. ISBN 0-471-49573-5- "The viability of thermal energy storage", Kamil Kaygusuz, Energy Sources, 21, 745-755, (1999).

(5) Domínguez M., Pinillos J. M, García C., Gutiérrez P. Sistema pasivo de climatización: CSIC. España, 18 Mar 1999 Patente de invención. no 9900558.

(6) Feldman, D., Banu D., Hawes D.: Low chain esters of stearic acid as phase change materials for thermal energy storage in buildings, Solar Energy Materials and Solar Cells (1995), pp. 311-322. http://dx.doi.org/10.1016/0927-0248(94)00186-3

(7) Hart, H., Hart. D. J., Craine. L. E.: Organic Chemistry - A Short Course, Ninth Edition, Houghton Mifflin Company (1995).

(8) Hasan, A., Sayigh, A., Some Fatty Acids as Phase Change Thermal Energy Storage Materials. Renewable Energy, (1994), Vol. 4, No 1, pp. 69-76. http://dx.doi.org/10.1016/0960-1481(94)90066-3

(9) Hawes, D. W., Feldman, D.: Absorption of phase change materials in concrete, Solar Energy Materials and Solar Cells 27 (1992), pp. 91-101. http://dx.doi.org/10.1016/0927-0248(92)90112-3

(10) Hawes, D. W., Feldman, D., Banu, D.: Latent heat storage in building materials, Energy and Buildings, 20 (1993), pp. 77-86. http://dx.doi.org/10.1016/0378-7788(93)90040-2

(11) Manz, H., Egolf, P. W., Suter, P., Goetzberger, A.: TIM-PCM External Wall System for Solar Space Heating and Daylighting, Solar Energy 61 (1997), pp. 369-379.

(12) Scalat, S., Banu, D., Hawes, D., Paris, J., Haghighata, F., Feldman, D.: Full scale thermal testing of latent heat storage in wallboard, Solar Energy Materials and Solar Cells, 44 (1996), pp. 49-61. http://dx.doi.org/10.1016/0927-0248(96)00017-7

(13) Setterwall, F.: (2000). Annex 10 - Phase change materials (PCM) and chemical reactions for thermal energy storage (TES). Proc. TERRASTOCK 2000, 8th International Conference on Syed M et al., Thermal Storage Using Form Stable Phase Change Materials, ASHRAE Journal, 36 (1997), pp. 45-50. 